\title{
Raumstrategien privater Akteure
}

\author{
Das BID Reeperbahn+ und die Kampagne „St. Pauli pinkelt zurück“
}

\section{„Hier nicht pinkeln. Wir pinkeln zurück! Dein St. Pauli“}

Mit dieser Ansage hat sich das BID[1] Reeperbahn+, eine Interessenvertretung von Gewerbetreibenden und Grundeigentümer_innen auf St. Pauli, im Frühjahr 2015 international Gehör verschafft. Der Satz steht auf dort angebrachten Warnschildern, denn die Kampagne „St. Pauli pinkelt zurück“ richtet sich an die Gäste und Besucher_innen der Reeperbahn und bezieht sich auf deren Urinierverhalten, welches diese Maßnahme aus BID-Perspektive nötig erscheinen ließ. Aus diesem Grund wurden vom BID Reeperbahn+ besonders oft von Wildpinkler_innen heimgesuchte Wände mit einem unsichtbaren, superhydrophoben Lack beschichtet. Diese Maßnahme hat zum Ziel, dass künftige Wildpinkler_innen ihren Urin überraschend selbst abbekommen: Der Lack verändert die Oberflächenstruktur und lässt den Urin wie von einer Lotusblüte abprallen - so pinkelt St. Pauli zurück. Begleitet wurde diese Kampagne von einem Video[2], das auf Youtube mittlerweile über 5 Millionen Klicks erreicht hat. Auch in der internationalen Berichterstattung war das Medienecho groß.

Das BID Reeperbahn+ setzt also als reichweitenstarker Akteur das Wildpinkeln auf die Agenda. Doch was ist mit dieser Kampagne genau beabsichtigt? Geht es nur darum, eine Ordnungswidrigkeit zu verhindern? Wie präsentiert sich das BID Reeperbahn+ und wie versucht es seine Vorstellung von Ordnung im Raum St. Pauli durchzusetzen? Am Beispiel der Kampagne „St. Pauli pinkelt zurück“ soll dies erörtert werden. Dass das öffentliche Urinieren als Belästigung empfunden werden kann, steht hierbei außer Frage, aber der Fokus liegt auf der allgemeineren Botschaft des BID Reeperbahn+. Welche Vorstellung wird in der Kampagne an wen herangetragen? Welche Interessen leiten die Akteure eines BID, wenn es um die Grenzziehung zwischen angemessenem und nicht angemessenem Verhalten geht, und welche Sprache wählen sie, um diese Grenze aufzuzeigen? 


\section{St. Pauli und sein Image}

St. Pauli hat den für Gentrifizierungsprozesse typischen Wandel vom Arbeiterquartier und als sozialer Brennpunkt bezeichnetem Ort zum Szeneviertel durchlaufen. St. Pauli gilt als divers und kreativ. Der Stadtteil ist seit einigen Jahren einem enormen Veränderungsdruck ausgesetzt und hat sich, neben seiner Funktion als Vergnügungsviertel, zu einem Hotspot der Kreativszene entwickelt. Der Hamburger Stadtteil befindet sich im Bezirk HamburgMitte und ist ein dicht besiedeltes Wohngebiet im Innenstadtbereich. 2012 lebten dort 21.741 Menschen. Bei einer Fläche von 2,3 Quadratkilometern beträgt die Zahl der Einwohner_innen pro Quadratkilometer 9437 und liegt damit deutlich höher als der Durchschnitt des Bezirks Hamburg-Mitte von 2135 Personen pro Quadratkilometer (vgl. Statistisches Amt für Hamburg und Schleswig-Holstein 2014). Neben seiner Funktion als Wohngebiet ist St. Pauli vor allem als Kultur- und Vergnügungsviertel bekannt und somit durch seine zahlreichen Besucher_innen geprägt. Mit der Reeperbahn sowie dem Hafenrand ist St. Pauli nicht nur für Hamburger_innen ein Ort für Freizeitaktivitäten, sondern auch ein beliebtes Ziel von Tourist_innen. Besonders die Vermarktung der Reeperbahn als die ,sündigste Meile der Welt' machte den Stadtteil weit über die Grenzen Hamburgs hinaus bekannt. In einer Studie mit dem Titel „Kreative Milieus und offene Räume in Hamburg" bezeichnet die Hamburger Behörde für Stadtentwicklung und Umwelt St. Pauli zudem als „Kreative Stammzelle Hamburgs“ (BSU Hamburg 2010: 114).

Das war nicht immer so. Die historische Entwicklung zum Vergnügungsviertel ist dadurch beeinflusst, dass St. Pauli lange Zeit vor der Stadtmauer Hamburgs lag. In St. Pauli konnten sich Gewerbe ansiedeln, die in Hamburg nicht erwünscht waren. Auch verarmte Menschen, die sich Ende des 18. Jahrhunderts aufgrund steigender Mieten keine Bleibe mehr in Hamburg leisten konnten, zogen vermehrt in die Vorstadt (vgl. Manos 1989: 86). Die Gemengelage aus verarmter Sozialstruktur und zwielichtigem Vergnügungsviertel führte zur Stigmatisierung des Stadtteils. Die Ansiedlung des Rotlichtmilieus begünstigte dies. Manos beschreibt, dass auch nach der Eingemeindung St. Paulis eine „,moralische‘ Grenze zu Sankt Pauli - ein grobgesponnenes Geflecht aus Angst und Faszination, Begierde und Verachtung“ - bestehen blieb (ebd.: 89). Die Diffamierung wurde zur Werbung, als Hamburger Geschäftsleute Mitte des 19. Jahrhunderts das Potential des Vergnügungsviertels erkannten, so Manos (ebd.:116). Das Image des sündigen Viertels wurde forciert und stellt bis heute einen Standortfaktor dar. Manos führt weiter aus, dass die „Politik der Ausbeutung und Ausgrenzung“ die Beziehung der Bürger_innen St. Paulis „zur ,Mutterstadt“ Hamburg geprägt" habe und spricht ihnen aufgrund der vom Widerstand geprägten Lebensbedingungen „Konfliktfähigkeit und bedingte Solidarität“ zu (ebd.). Auch die Zuschreibung des, widerspenstigen St. Pauli' bestimmt bis heute das Image des Viertels.

Der ehemals marginalisierte Stadtteil erfährt seit einigen Jahren also einen Wandel, und das kreative Image gehört mittlerweile zum kulturellen Kapital St. Paulis, was Gentrifizierungsprozesse anstößt. In seinem Blog beschreibt Andrej Holm, dass Gentrifizierung ,immer auch ein Prozess der 
symbolischen Aufwertung des Ortes und der Kommodifizierung kulturellen Kapitals ist" (Holm 2013). Für Hamburg ist St. Pauli also wichtig, weil es sich um eine Touristenattraktion und um einen Imagefaktor für die Stadt Hamburg handelt.

Um diesen zu bewahren, hat sich das BID Reeperbahn+ dazu entschieden, einen Claim zu entwickeln: „Your pleasure, our home“. Dieser soll darauf aufmerksam machen, dass St. Pauli zwar sündige Vergnügungsmeile ist, aber dort auch Menschen wohnen, auf die es Rücksicht zu nehmen gilt. In diesem Claim enthalten ist die Aufteilung zwischen,Wir' und ,Ihr. Diese Aufteilung überträgt sich nun von der sozialen auch auf die räumliche Ebene der ,Vergnügungsmeile und des ,Zuhauses“ und vermittelt, dass beides schwer zusammengeht. Die Grenze wird also nicht nur bezüglich eines Verhaltens aufgezeigt, sondern auch bezüglich sozialer Gruppen, in diesem Fall Bewohner_innen versus Besucher_innen. Bei dem angestrebten Ziel, den Gästen wichtige Grenzen aufzuzeigen, muss das BID Reeperbahn+ eine Gratwanderung bestehen. Einerseits lautet das ausgewiesene Ziel: „Sankt Pauli bleibt frei, bunt und tolerant. Denn Sankt Pauli war immer offen für alle Kulturen dieser Welt.“ (Pfadt 2016) Gleichzeitig soll dabei die als Vorteil empfundene „St.Pauli-typische Nutzungsmischung“ erhalten bleiben (Pfadt 2013).

\section{Das BID Reeperbahn + pinkelt zurück - die Kampagne}

Die Initiative für das BID Reeperbahn+ ging von den Grundeigentümer_ innen und der Interessengemeinschaft St. Pauli und Hafenmeile e.V. aus. Die IG St. Pauli und Hafenmeile e.V. besteht seit 1985 und sieht sich selbst als

Abb. 1 Gebietsabgrenzung des BID Reeperbahn+ (Quelle: Pfadt 2013) Interessenverband für die in St. Pauli ansässigen Unternehmen und Betriebe, der durch seine Aktivitäten das wirtschaftliche und soziale Geschehen im Stadtteil beeinflusst. Nach eigener Aussage steht dabei die „Entwicklung als touristischer Standort unter Berücksichtigung des eigenwilligen Charme[s]

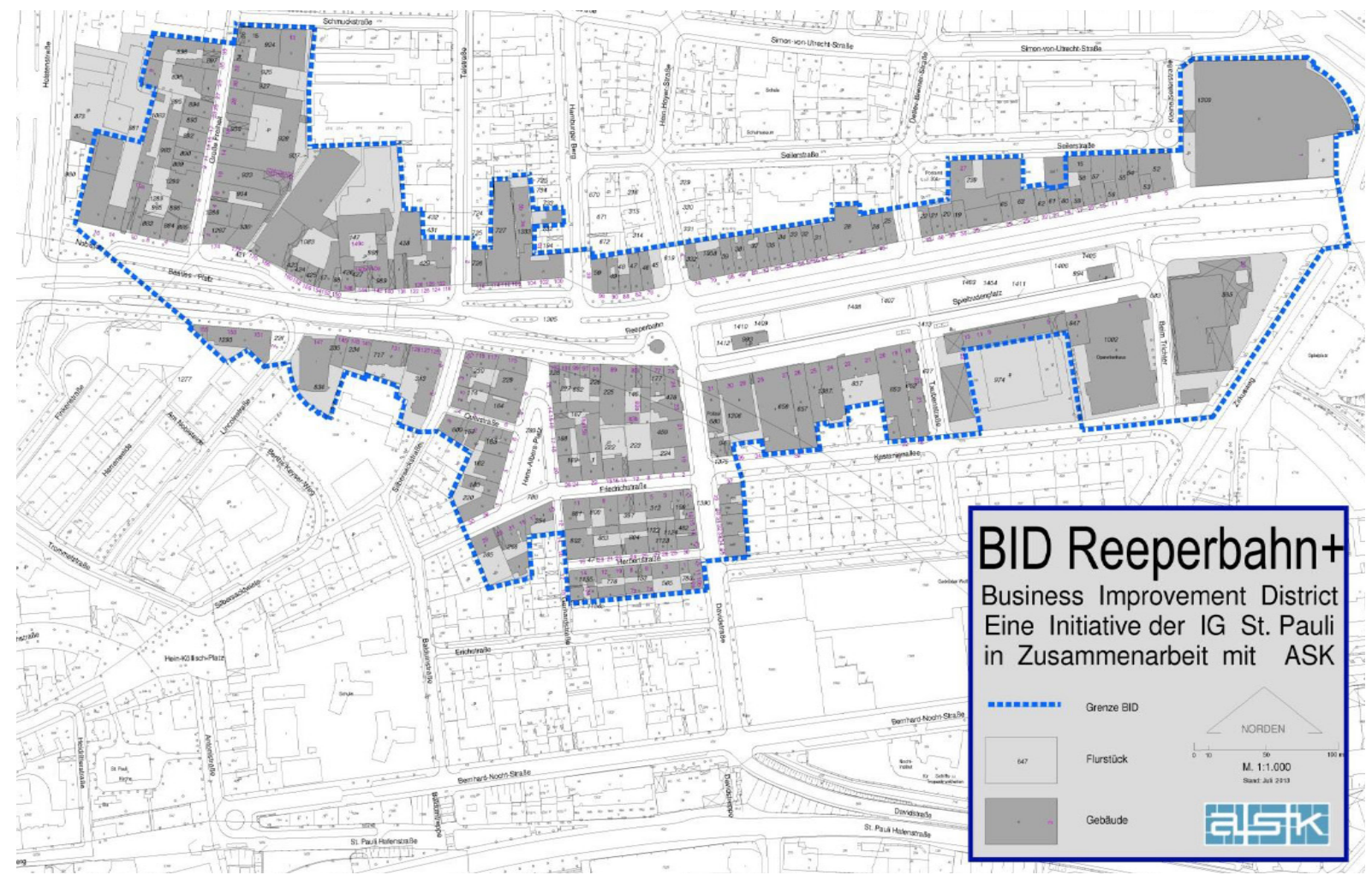


und Flair[s] St. Paulis“" (Pfadt 2016) im Fokus. Das Budget für die fünfjährige Laufzeit des BID beläuft sich auf insgesamt 1.890.375 Euro. Über eine Abgabe, die alle BID-,,Teilnehmer_innen“ zu zahlen verpflichtet sind, finanziert sich ein BID. Wie hoch genau diese ausfällt, unterscheidet sich von Fall zu Fall.

Für die Entwicklung und Koordinierung der Maßnahmen in Hamburg wurde die ASK Hassenstein+Pfadt GmbH ausgewählt. Das Quartiersentwicklungsbüro schlug zum Beispiel vor, die Reeperbahn mit Infopoints in der Form von Frauenkörpern zu bestücken, eine Reeperbahn-Marke zu erschaffen, ähnlich dem „Welcome-to-Fabulous-Las-Vegas“-Schild, oder auch sogenannte,waste watchers' einzusetzen - eine Art Ordnungsdienst, der für Sauberkeit sorgen soll und befugt ist, bei Verunreinigungen ein Strafgeld zu verhängen.

In dem Statement „Wir sind hier nicht in Disneyland!“von S.O.S. St. Pauli, einem Bündnis, das Mitbestimmung der Bevölkerung in der Stadtentwicklungspolitik fordert, wurden im Vorfeld Bedenken zur Realisierung eines BID um die Reeperbahn herum geäußert (Jörg 2013). Generell wird das Antreiben des Gentrifizierungsprozesses kritisiert, der Mietsteigerungen im angrenzenden Gebiet zur Folge hat. Ebenso besteht die Gefahr der Vertreibung jahrelang ansässiger Geschäftsinhaber_innen auf Grund der verpflichtenden Abgabe. Außerdem wird wegen der potentiellen Aussprache von Platzverweisen bei nicht erwünschtem Verhalten im Sinne der Gewerbetreibenden mit dem Verschwinden des „üblichen“ Klientels gerechnet, beispielsweise Anwohner_innen oder Nicht-Konsument_innen. Herr Pfad, Leiter des ASK, nimmt dazu im BID-Antrag Stellung:

„Dieser Strukturwandel verläuft keineswegs problemlos und erzeugt, wie alle vergleichbaren Stadtentwicklungsprozesse, Gewinner und Verlierer. Er birgt die Gefahr, dass die Elemente des traditionellen Vergnügungsviertels allmählich verschwinden, die das besondere Flair und Milieu dieses Stadtteils ausmachen. Ziel verstärkter Anstrengungen, in diesen Wandlungsprozess einzugreifen, ist es nicht, ihn abzubremsen oder zu verhindern, sondern seine Entwicklungsrichtung nach Möglichkeit so zu beeinflussen, dass der Stadtteil insgesamt und vor allem der Bereich des Vergnügungsviertels, wie er jetzt als BID-Gebiet ausgewiesen ist, davon profitieren. Aus Besuchern sollen Kunden gemacht werden.“ (Pfadt 2013).

Der Quartiersmanager findet, dass der Begriff ,Gentrifizierung' nicht den Prozess beschreibe, dem St. Pauli unterliegt; er nennt es ,Strukturwandel'. Dieser bewirke einen Wandel in der Angebots- und Standortstruktur von Einzelhandel, Dienstleistungen und Kulturangeboten. Welche Elemente durch den ,Strukturwandel' gefährdet werden, wird nicht genau benannt. Dadurch, dass darauf hingewiesen wird, dass eben diese Elemente das ,Flair' und ,Milieu' ausmachen, wird aber durchaus suggeriert, dass das Image St. Paulis in Gefahr sei. Das BID Reeperbahn+ nutzte außerdem für seinen Antrag Zahlen und Formulierungen einer Strukturbefragung der Handelskammer Hamburg aus dem Jahr 2012. Die Ergebnisse aus der unter Gewerbetreibenden und Besucher_innen St. Paulis durchgeführten Befragung besagen unter anderem, dass 90 Prozent der Gewerbetreibenden Sauberkeit für wichtig oder sehr wichtig betrachten und dabei nur 11 Prozent 
die Sauberkeit als gut oder sehr gut beurteilten. Gleichzeitig seien zwei Drittel der Besucher_innen mit der Sauberkeit in St. Pauli unzufrieden. Die Ableitungen aus diesem empirischen Material spiegeln sich bei Handelskammer und BID Reeperbahn+ in der Formulierung: „Nicht die Sicherheit, sondern die mangelnde Sauberkeit ist aus Sicht der Unternehmen und der Besucher die Achillesferse des Stadtteils“ (Pfadt 2013). Die Investition in den wasserabweisenden Lack sowie die Produktion und Verbreitung des dazugehörigen Videos schienen daher offensichtlich nötig.

Zeit, sich das millionenfach verbreitete Werk genauer anzuschauen. Die bildliche Ebene des Videos aus der Kampagne soll hier zwar nicht intensiv analysiert werden, die ausgewählten Bilder vermitteln aber insgesamt einen bedrohlichen Eindruck, da vermehrt junge Männer in Dunkelheit mit aufgezogenen Kapuzen gezeigt werden, die sich eine Wand zum Bepinkeln suchen und dann ,zurückbepisst‘ werden. Dazu ist Folgendes zu hören:

„Sprecher: St. Pauli ist das Partyviertel in Hamburg und für über 20 Millionen Besucher im Jahr ein Riesenspaß. Für die Leute, die hier wohnen, ist es nicht immer so lustig. Denn Betrunkene pinkeln überall hin und ruinieren das Stadtbild.

Person 1 (älterer Mann): Es pisst mich tierisch an, dass hier überall Leute hinpissen, das nervt!

Person 2 (junge Frau): Und vor allem nervt es, wenn auf die Kinderspielplätze gepisst wird.

Person 3 (Frau mit Kind): Das Gepinkel an die Wände in unserem Stadtteil nervt. Lasst es einfach!

Sprecher: Verbote oder Bußgelder bringen kaum etwas. Darum lösen wir das Problem jetzt auf unsere Art. St. Pauli pinkelt zurück! (Es ist zu sehen, wie einige der Schilder aufgehängt werden, auf denen steht: ,DO NOT PEE HERE! We pee back!') Wir haben im ganzen Viertel oft heimgesuchte Wände mit einem hydrophoben Lack beschichtet. Dieser Lack ist so wasserabweisend, dass Flüssigkeit von der Oberfläche regelrecht abprallt. Damit machen wir unsere Botschaft nicht nur allen klar, sondern stellen auch sicher, dass sie bei unserer Zielgruppe ankommt.

Julia Staron (Quartiersmanagerin des BID Reeperbahn+): Mittlerweile sind viele von den Wänden mit dem Lack versehen und man kann jetzt nur sagen: Liebe Wildpinkler seid gewarnt, denn nicht jede Wand trägt auch den Hinweis darauf. (Mit Zeigefinger und Blick auf die Kamera gerichtet:) Seht euch vor! St. Pauli pinkelt ab sofort zurück!“ (BID Reeperbahn 2015, Transkription FH)

Deutlich wird in diesem Video, wie die direkte Ansprache an die Wildpinkler erfolgt: auf ,unsere Art‘. Die Sprache vermittelt, dass es um ein ernstes, problematisches Verhalten von Besucher_innen geht, welches, von uns' nicht länger akzeptiert wird. Man will zwar nicht auf die Finger hauen, aber man pinkelt zurück. Das soll frech wirken und damit die anvisierte Strategie der Erziehung mit einem Augenzwinkern versehen. An dieser kommunikativkreativen Kampagne des BID Reeperbahn + zeigt sich, wie versucht wird, die 
Maßnahme st.-pauli-gemäß zu verpacken. Sich über die Diversität, das Flair und die Bedeutung ebendieses Raumes für Hamburg bewusst, nimmt sich das BID Reeperbahn+ das Recht, als St.-Pauli-Repräsentant für Sauberkeit und Ordnung zu werben, und zwar ohne dass dies wie Repression wirkt, um das positive Image des Stadtteils zu wahren.

Ein kriminologisch sehr bekanntes Prinzip wird dabei für die Kampagne verwendet: das Mittel der Abschreckung. Der superhydrophobe Lack stellt zwar das Zentrum der Kampagne dar, und auf seiner Funktion basiert die ,freche Art der Kommunikation, aber tatsächlich spielt er keine Rolle, denn man kann nicht wissen, ob der ziemlich teure Lack tatsächlich von BIDMitarbeiter_innen auf die Wände St. Paulis aufgetragen wird, beziehungsweise auf wie viele oder welche. Offizielle Informationen hierzu werden nicht herausgegeben, und dies ist wenig verwunderlich. Was ist, wenn die Tourist_innen sich dieses Video nicht angucken, trotzdem gegen die Wand pinkeln und nichts passiert?

Lediglich die Schilder, die vorgeben, dass Vorsicht zu wahren sei, weil zurückgepinkelt werden könnte, sollen eine Gefahr vermuten lassen. Damit wird bei dieser Maßnahme nicht nur mit dem Prinzip der Abschreckung gespielt, sondern es wird darüber hinaus das Prinzip der panoptischen Überwachung bedient, da es sich um nicht erkennbaren Klarlack handelt. Dadurch, dass die Quartiersmanagerin warnt, dass nicht alle Wände mit dem entsprechenden Schild versehen sind, lauert die potentielle Selbstbepinkelungs-Gefahr damit überall und man kann nicht wissen, ob man durch die Abweichung bestraft wird. Der Panoptismus findet sich insofern hier als Kontrollmechanismus wieder.

Weit über seine Gebietsgrenzen hinaus konstruiert das BID Reeperbahn+ mit dieser (Kommunikation-)Strategie den Raum St. Pauli und seine ,Probleme' und die scheinbar angemessene Reaktion darauf. Dabei ist es nicht St. Pauli, sondern das BID, das zurückpinkelt. Bei der Konstruktion des Problems Wildpinkeln bezieht das BID aber auch die Bewohner_innen St. Paulis mit ein, obwohl es für diese kein legitimes Sprachrohr darstellt und sie auch nicht bei der Strukturbefragung, auf die das BID sich stützt, berücksichtigt wurden. Der Absender des Slogans „St. Pauli pinkelt zurück“ wird aktiv verschleiert, und durch Werbesprache wird ein ,normales' Bild generiert, das die Fakten über die Veränderung und Teilhabe am öffentlichen Raum verwischt.

Die Hamburger Morgenpost berichtete in Reaktion auf die Kampagne sogar, dass die Anwohner_innen nichts von der Anti-Pinkler-Aktion gewusst hätten und ihr skeptisch gegenüber stünden (vgl. Schlink 2015). Der in dem Artikel zu Wort kommende Steffen Jörg von der GWA St. Pauli vermutet hinter der Kampagne das fragwürdige Ziel, den Tourismus im Stadtteil weiter zu stärken: „Der BID fördert den zusätzlichen Ansturm auf den Kiez und tut nun so, als würde man sich um die von ihnen verursachten negativen Folgen kümmern." (zit. nach Schlink 2015)

Es ergibt sich also ein komplexes Netz von Akteuren und deren Interessen. Was das BID mit seiner Kampagne für ein Interesse verfolgt, wird hier aber

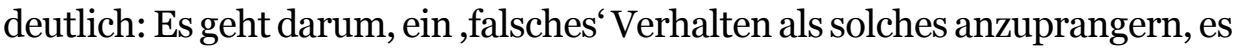
zu verdrängen und somit gleichzeitig die als soziale Gruppe definierten, Wildpinkler' aus dem BID-Gebiet zu exkludieren. Diese Macht zur Distinktion 
kann also nicht nur räumliche und wirtschaftliche Konsequenzen haben, sondern vor allem auch soziale. Das Verhalten soll, entgegen der imaginierten Tradition des Stadtteils, normiert werden. Das Alleinstellungsmerkmal frei zu sein auf der Reeperbahn, wird mit einem Erziehungsanspruch gemaßregelt, und dies aus einem wirtschaftlichen Interesse heraus. Betroffen davon sind sowohl Bewohner_innen als auch Besucher_innen und Gewerbetreibende des Gebiets. Anknüpfend an die vorherigen Ausführungen von Manos zur moralischen Grenze kann hier der von Sharon Zukin geprägte Begriff der ,moral landscape herangezogen werden. Dieser kann so verstanden werden, dass ein Raum durch dynamische Handlungsmuster seiner kulturell agierenden Akteure definiert wird: „Raum strukturiert auch die menschliche Wahrnehmung, die Interaktionen und das Gefühl von Wohlbefinden oder Verzweiflung, Vertrautheit oder Fremdheit.“ (Zukin 2013: 361) Ähnliches meint Guido Lauen mit der ,Rhetorik der Ausgrenzung': „Sich abweichend zu verhalten verweist angesichts der Wahrheit und Machtwirkungen des kriminologischen Diskurses auf die mangelhafte moralische Integrität des Abweichenden als Verstoß gegen die hegemoniale Vorstellung über das, was richtig und falsch in einer Gesellschaft ist." (Lauen 2011: 146) Das sündige, widerspenstige Flair wird als rhetorisches Mittel vom BID Reeperbahn+ aufgenommen, um inhaltlich zu vermitteln, dass beim Wildpinkeln eine moralische Grenze überschritten wird.

\section{Die Unternehmerische Stadt}

„Unternehmen Hamburg“ ist Teil einer berühmt gewordenen Rede, die Klaus von Dohnanyi, der damalige Bürgermeister Hamburgs, im Jahre 1983 gehalten hat. In Anbetracht des Niedergangs der Hafenökonomie forderte Dohnanyi eine Wirtschaftsförderungspolitk, die es Hamburg ermögliche, im Wettbewerb mit anderen Städten zu bestehen (vgl. Ronneberger et al. 1999: 30). Die Rede gilt als Meilenstein der neoliberalen Stadtpolitik, da Dohnanyi der erste deutsche Landes- und Kommunalpolitiker war, der das Gemeinwesen Stadt mit einem profitorientierten Unternehmen gleichsetzte (ebd.). Neoliberale Entwicklungsstrategien haben in Städten zur Folge, dass Markt- und Wettbewerbsmechanismen auf die interne Steuerung städtischer Prozesse übertragen werden (vgl. ebd). So werden etwa künstlich Wettbewerbsverhältnisse geschaffen, indem die städtische Infrastruktur privatisiert wird. Besonders Stadtzentren sollen der Öffentlichkeit als Visitenkarte der Stadt präsentiert werden, wodurch eine urbane Kultur zu einer der wichtigsten Kapitalanlagen wird. „Die Unterhaltungsindustrie und der Einzelhandel versuchen wiederum, für diese Formen der Identitätsbildung die notwendigen Symbole und Zeichen mitzuliefern, indem sie die Waren und Dienstleistungsprodukte als ,Erlebnis“ oder ,Lifestyle“ anbieten.“ (vgl. ebd.: 30) Mit diesen veränderten Alltagspraxen geht auch eine systematische Funktionalisierung der Raumgestaltung einher. „Überall entstehen neue kommerzielle Einrichtungen, die die Bereiche Konsum, Freizeit und Entertainment miteinander verbinden." (ebd.) St. Pauli, speziell die Reeperbahn, stellt eine solche kommerzielle Erlebniswelt dar und bedient damit den Idealtypus eines öffentlichen Raumes, der scheinbar den vorherrschenden Alltags- und Konformitätsnormen entgegensteht. 
Durch eine solche Ausrichtung auf Konsum- und Erlebnislandschaften intensiviert sich notwendigerweise die politische Administration des öffentlichen Raumes, die sich vor allem gegen die sichtbare Anwesenheit von Marginalisierten an zentralen Orten und Plätzen richtet (vgl. ebd.). Konsumabträgliche Situationen sollen zum Beispiel durch die Installierung von Überwachungstechnologien vermieden werden. Da solche Präventionsstrategien durch profitorientierte Geschäftsleute befördert werden, liegen diesen privaten Konzepten auch deren Vorstellungen von abweichendem Verhalten zugrunde. Das kann bedeuten, dass schon Handlungsweisen ins Blickfeld geraten, die, devianzbegünstigend' sind, und nicht erst tatsächliche Straftaten.

Die Unternehmerische Stadt, die Standortlogik und der Städtewettbewerb führen also zur Aufwertung und Umstrukturierung der Innenstädte zu Erlebniswelten. Und die Absicherung dieser Konsumlandschaften begünstigt Strategien der Exklusion und Kontrolle.

\section{Der BID als neoliberales Instrument der Stadtentwicklung}

Basierend auf dem Paragraphen 171f BauGB sowie dem Gesetz zur Stärkung der Einzelhandels- \& Dienstleistungszentren (GSED), das 2005 in Hamburg eingeführt wurde, besteht die Möglichkeit, Business Improvement Districts (BIDs) zu gründen. Die Laufzeit eines BIDs ist auf fünf Jahre begrenzt, innerhalb derer Gewerbetreibende privat initiiert bestimmte städtische Aufgaben übernehmen. Ziele eines BID sind die grundlegende Aufwertung des Quartiers sowie eine Attraktivitätssteigerung des Gebietes im Verhältnis zur (städtischen) Umgebung. Die dazu verwendeten Maßnahmen werden aus Sicht der Gewerbetreibenden umgesetzt (vgl. Pfadt 2016). Als Bund Gewerbetreibender und Grundeigentümer_innen sowie durch ihre staatlich erteilte Handlungskompetenz tragen BIDs mutmaßlich zur (Teil-)Privatisierung des öffentlichen Raumes bei und gestalten ihn durch ihre Maßnahmen um. Nun kommt es natürlich darauf an, in welcher Art und Weise sie dies tun. „Im Prinzip geht es dabei um die Frage, wer mit welchen Mitteln die Macht in einer Stadt und/oder einem Quartier (tatsächlich) ausübt und damit die Geschicke der Quartiersentwicklung bestimmt." (Schnur 2014: 30) Mit der Kampagne „St. Pauli pinkelt zurück“ zeigt das BID Reeperbahn+ seine Ausrichtung als Ordnungsmacht, da es darum geht, ein bestimmtes Verhalten, das im Wildpinkeln besteht, im eigenen Sinne zu regulieren.

Hier kommt der Begriff ,Governance ins Spiel, welcher ebensolche Steuerungs- und Regulierungsmechanismen zusammenfasst. Es geht bei diesem Ansatz darum, wie verschiedene Akteursgruppen über die öffentliche Hand hinaus zusammenspielen und nebeneinander bestehen. Hierbei sind Schlüsselressourcen und damit Handlungsfähigkeiten für die Regelung gesellschaftlicher Sachverhalte an mehrere Akteure verteilt und unterliegen fortwährender Dynamik (vgl. Altrock/Bertram 2012: 9). Detlef Sack beschreibt das Phänomen der governance genauer als die Verlagerung von Kompetenzen und Ressourcen. Sie bedeute, ,dass auf einer politisch-administrativen Ebene Problemdefinitionen, Entscheidungen und deren Realisierung nicht allein durch staatliche, sondern zunehmend auch für nichtstaatliche Akteure erfolgen“ (Sack 2014: 92). Die Schlüsselressource des BID Reeperbahn+ 
liegt in der Kommunikation. Die Handlungsfähigkeit, mit dieser Ressource ein bestimmtes gesellschaftliches Problem zu regulieren, erhielt das BID Reeperbahn+ mit dem Zuschlag zur Gründung. Mit der Kampagne „St. Pauli pinkelt zurück“ bestimmt das BID Reeperbahn+ Wildpinkeln als nicht zu duldendes Problem und nutzt seine entsprechenden Mittel, um Meinung und Verhalten diesbezüglich in diesem Raum zu regulieren und zu beeinflussen. Diese Dynamiken und die hohe Medienwirksamkeit der Kampagne verweisen auch auf die Relevanz des Diskurses in dieser Sache. Dadurch, dass das BID das Wildpinkeln als Problem definiert, ergibt sich gleichzeitig die Chance, im Diskurs um Sauberkeit und Ordnung auf St. Pauli ein eigenes Deutungsmuster der Realität zu vermitteln und damit ein Bild (sozialer) Ordnung herzustellen.

Ist das Strategie? Laut dem Geographen Bernd Belina liegt eine strategische Praxis vor, „wenn das Subjekt sich, um den Gegenstand der Bedürfnisbefriedigung zu erhalten, notwendig gegen Andere durchsetzen muss (bzw. sich so verhält, als müsse es dies)." (Belina 2006: 50.) Dies ist beim BID und den von ihnen angesprochenen Wildpinklern insofern der Fall, als mit ihrer Maßnahme notwendigerweise zwei Kategorien gebildet werden: ,Wir' (St. Pauli) und ,Ihr' (die Wildpinkler). Es wird auch daran erkennbar, dass Anwohner_innen oder Nicht-Konsument_innen entsprechend der Grundidee eines BID nicht als Akteure einbezogen sind. Die Sprache der Kampagne wird aber trotzdem so gewählt, dass vermittelt wird, es spreche, ganz St. Pauli'. Die Ansprache bezieht sich dabei gezielt auf Gäste/Konsument_innen beziehungsweise Besucher_innen. Damit wird die binäre Trennung zwischen,Wir/St. Pauli und,Ihr/Gäste noch deutlicher. Es ist paradox: Die Sprache wird so gewählt, dass ein Gemeinschaftsgefühl entstehen soll, und doch zeigt sie gleichzeitig auf, dass man, um zur Gemeinschaft zu gehören - oder zumindest nicht beim Wildpinkeln bestraft zu werden -, sich der Ordnungsvorstellung des BID entsprechend verhalten muss. Das Flair des Stadtteils wird so in der Sprache aufgegriffen, aber mit dem inhaltlichen Appell wieder ausgehebelt. Auf die eingangs gestellte Frage, wie das BID es schafft, ein Bild von Ordnung durchzusetzen und gleichzeitig dem Image St. Paulis gerecht zu werden, kann nun also gesagt werden, dass es eben keine neuen öffentlichen Toiletten baut, sondern ein Youtube-Video produziert. Darin wird die Durchsetzung einer Konformitätsnorm als lustig verpackt und als stadtteilentsprechend verkauft. Durch das Anbringen des Lacks an öffentlichen Wänden wird zudem bereits devianzbegünstigendes Verhalten kontrolliert. Die Sicherheitsvorstellungen des BID werden dadurch sehr subtil implementiert. Schliesslich soll die Abschreckung ja nicht für den Konsum gelten, sondern eben nur fürs Pinkeln.

\section{Endnoten}

[1] $\quad \mathrm{BID}=$ Business Improvement District

[2] https://www.youtube.com/watch?v=uoN5EteWCH8\# (letzter Zugriff am 28.3.2018). 


\section{Autor_innen}

Friederike Häuser ist Studentin der Internationalen Kriminologie in Hamburg. Ihre Schwerpunkte sind Kontrollpraktiken und kritische Stadtforschung. Aktuell schreibt sie an ihrer Masterarbeit zum Thema Bodycams bei der Polizei.

ikehaeuser@gmx.de

\section{Literatur}

Altrock, Uwe / Bertram, Grischa (2012): Wer entwickelt die Stadt? Geschichte und Gegenwart lokaler Governance. Akteure - Strategien - Strukturen. Bielefeld: transcript.

Belina, Bernd (2006): Raum, Überwachung, Kontrolle. Vom staatlichen Zugriff auf städtische Bevölkerung. Münster: Westfälisches Dampfboot.

Behörde für Stadtentwicklung und Umwelt Hamburg (BSU) (2010): Kreative Milieus und offene Räume in Hamburg. Hamburg: Behörde für Stadtentwicklung und Umwelt.

BID Reeperbahn (2015): St. Pauli pinkelt zurück. St. Pauli Peeback. https://www.youtube. com/watch?v=uoN5EteWCH8 (letzter Zugriff am 14.8.2017).

Holm, Andrej (2013): Wir bleiben Alle! Münster: Unrast.

Jörg, Steffen (2013): Statement von S.O.S. St. Pauli zum geplanten Busines Improvement District (BID) Reeperbahn. Wir sind hier nicht Disneyland. http://www.sos-stpauli. de/statement-von-s-o-s-st-pauli-zum-geplanten-busines-improvement-district-bidreeperbahn/ (letzter Zugriff am 14.9.2017).

Lauen, Guido (2011): Stadt und Kontrolle. Der Diskurs um Sicherheit und Sauberkeit in den Innenstädten. Bielefeld: transcript.

Manos, Helene (1989): Soziale Lagen und Soziale Fragen im Stadtteil Sankt Pauli. Hamburg: Ergebnisse Verlag.

Pfadt, Andreas (2013): Antrag für die Errichtung des Business Improvement Districts Reeperbahn+. https://www.hk24.de/blob/hhihk24/produktmarken/branchen/ handel/ downloads/1151808/5a604d610f7bf4245f52d4d27e8aa442/Antrag_zur_Gruendung_ eines_BID_Reeperbahn-data.pdf (letzter Zugriff am 24.8.2017).

Pfadt, Andreas (2016): BID Reeperbahn - Ziele. http://bid-reeperbahn.de/ziele/> (letzter Zugriff am 18.09.17).

Ronneberger, Klaus / Lanz, Stephan / Jahn, Walther (1999): Die Stadt als Beute. Bonn: Dietz.

Sack, Detlef (2014): Governance in lokalen Räumen. In: Bernd Belina / Matthias Naumann / Anke Strüver (Hg.), Handbuch Kritische Stadtgeographie. Münster: Westfälisches Dampfboot, 92-96.

Schlink, Mike: (2015): Spezial-Lack auf St. Pauli. Anwohner wussten nichts von Anti Pinkler Aktion http://www.mopo.de/hamburg/spezial-lack-auf-st--pauli-anwohner-wusstennichts-von-anti-pinkler-aktion--1037474 (letzter Zugriff am 14.9.2017).

Schnur, Olaf (2014): Quartiersforschung im Überblick. Konzepte, Definitionen und aktuelle Perspektiven. Wiesbaden: Springer.

Statistisches Amt für Hamburg und Schleswig-Holstein (2014): Hamburger Stadtteil-Profile. www.statistik-nord.de/uploads/tx_standocuments/NR13_Stadtteil-Profile_2012.pdf (letzter Zugriff am 23.9.2017).

Zukin, Sharon (2013): Moralische Landschaften. In: Susanne Hauser / Christa Kamleithner / Roland Meyer (Hg.), Grundlagentexte aus den Kulturwissenschaften. Bielefeld: transcript, 354-365. 